\title{
Charge Quantization from a Number Operator
}

\author{
Cohl Furey* \\ Perimeter Institute for Theoretical Physics \\ E-mail: cfureyaperimeterinstitute.ca
}

In the early seventies, Günaydin and Gürsey discovered $S U_{c}(3)$ quark structure in the split octonions, [1]. Using their anti-commuting ladder operators, $\alpha_{i}$, we show a direct route to a new $U(1)$ generator. This $U(1)$ generator behaves like electric charge, thereby allowing us to further identify states behaving like the electron and neutrino.

Our proposed electric charge turns out to be proportional to a number operator, consequently illuminating why it is quantized.

Using only this trio of ladder operators, and their conjugates, we construct a pair of minimal left ideals, which is shown to transform under $S U_{c}(3)$ and $U_{e m}(1)$ as does a full generation of the standard model.

References

[1] M. Günaydin, F. Gürsey, Quark Statistics and Octonions, Phys. Rev. D, Vol. 9, No. 12 (1974)

Frontiers of Fundamental Physics 14 - FFP14,

15-18 July 2014

Aix Marseille University (AMU) Saint-Charles Campus, Marseille

\footnotetext{
${ }^{*}$ Speaker.
} 